(C) 2020 IEEE. Personal use of this material is permitted. Permission from IEEE must be obtained for all other uses, in any current or future media, including reprinting/republishing this material for advertising or promotional purposes, creating new collective works, for resale or redistribution to servers or lists, or reuse of any copyrighted component of this work in other work

\title{
Effect of the Inner Current Loop on the Voltage Regulation for Three-Phase Photovoltaic Inverters
}

\author{
Andoni Urtasun, Pablo Sanchis, and Luis Marroyo \\ Department of Electrical, Electronic and Communication Engineering) \\ Institute of Smart Cities \\ Public University of Navarra (UPNA) \\ Pamplona, Spain
}

\begin{abstract}
In three-phase grid-connected PV inverters, regulating the input voltage is a fundamental requirement. In order to reduce the influence of the $P V$ non-linear behavior and ensure stability in the whole operating range, the input capacitance is currently oversized. This paper reveals the important effect of the inner current loop in the voltage stability and proposes to use a Proportional (P) controller instead of a PI controller. If tuned following the guidelines provided in this paper, the $P$ controller makes it possible to design a stable voltage loop without increasing the input capacitance, thus reducing the converter cost.
\end{abstract}

Keywords-control design, control stability, inner current loop, photovoltaic generator, three-phase inverter.

\section{INTRODUCTION}

In order to connect the photovoltaic (PV) generator to the grid, three-phase inverters with an LCL filter are frequently used, as shown in Fig. 1 [1]. With the purpose of controlling the PV generator operating point in this system, an input voltage regulation is carried out by means of a cascaded regulation, where the outer loop obtains the active current reference to be injected into the grid. The control scheme is shown in Fig. 1 for the particular case of a current control in dq reference frame with no feedforward compensations, where the superscript $*$ represents reference variable and the subscript $f$ represents measured and filtered variables. As can be observed, the $\mathrm{d}$ current is used to control the PV voltage while the q current makes it possible to set the reactive power injected into the grid.

The voltage regulation must be designed to remain stable in the whole operating range. However, it has been shown that the open-loop transfer function of the dc voltage regulation strongly depends on the operating point, and a Right-HalfPlane (RHP) pole appears when the PV voltage is below the Maximum Power Point (MPP) voltage [2], [3]. Although this operating point is not sought, the system can end up in this situation after an irradiance drop or a misled MPPT algorithm. In this case, in order to ensure stability, the crossover frequency should be set at least two times higher than the frequency of the RHP pole [4]. However, to reject the second harmonic in the dc voltage inherent to unbalanced grids (especially significant during asymmetric voltage sags), the crossover frequency is limited to $10-15 \mathrm{~Hz}$. For this reason, the input capacitor must often be oversized in order to reduce the RHP pole frequency and then guarantee voltage control stability [5].

To avoid oversizing the capacitor, it has been proposed to modify the voltage control so that a virtual impedance is emulated in parallel with the PV generator, making it possible to remove the RHP pole [3]. In this paper, a simpler solution is proposed to achieve the same objective. It is based on modifying the inner active current loop by using a Proportional (P) instead of a Proportional-Integral (PI) controller. If tuned following the guidelines provided in this paper, the P controller makes it possible to remove the RHP pole and thus to readily design a stable voltage control.

\section{MODELING AND CONTROL OF THE INVERTER}

\section{A. Open-Loop Representation}

The system is modeled in dq-reference frame after applying the power-invariant abc-dq transformation. Given that the RHP pole appears at low frequency, an ideal strong grid, i.e. $L_{g} \approx 0$, is assumed to avoid unnecessarily complicating the model. The average model is thus obtained as [5]

$$
\left\{\begin{array}{l}
L \cdot \frac{d i_{d}}{d t}=d_{d} \cdot v_{d c}+\omega_{0} L \cdot i_{q}-r_{L} \cdot i_{d}-v_{g d} \\
L \cdot \frac{d i_{q}}{d t}=d_{q} \cdot v_{d c}-\omega_{0} L \cdot i_{d}-r_{L} \cdot i_{q}-v_{g q} \\
C_{d c} \cdot \frac{d v_{d c}}{d t}=i_{p v}-d_{d} \cdot i_{d}-d_{q} \cdot i_{q}
\end{array}\right.
$$

where $i_{d}$ and $i_{q}$ are the inductor currents, $v_{g d}$ and $v_{g q}$ the grid voltages, $d_{d}$ and $d_{q}$ the duty cycles, $r_{L}$ the equivalent resistance in series with the inductors, and $\omega_{0}$ the grid angular frequency.

The steady-state operating point can be solved from (1) by setting the derivate terms to zero. Taking into account that the Phase-Locked Loop (PLL) synchronizes the d-axis with the grid voltage, one obtains

$$
\left\{\begin{array}{l}
D_{d}=\frac{V_{g}-\omega_{0} L \cdot I_{q}+r_{L} \cdot I_{d}}{V_{d c}} \\
D_{q}=\frac{\omega_{0} L \cdot I_{d}+r_{L} \cdot I_{q}}{V_{d c}} \\
I_{d} \approx \frac{V_{d c} \cdot I_{p v}}{V_{g d}}
\end{array}\right.
$$

The model shown in (1) is linearized by applying smallsignal variations (marked with a circumflex) around the steady-state operating point represented in (2). The PV current depends on the PV voltage through a nonlinear I-V curve and its linearization can be expressed as $\hat{\imath}_{p v}=-g_{p v} \cdot \hat{u}_{p v}$, where $g_{p v}$ is the PV dynamic conductance, equal to the absolute value of

This work was supported by the Spanish State Research Agency (AEI) under grant PID2019-1 10956RB-I00/AEI/10.13039 and grant DPI-201680641-R. 

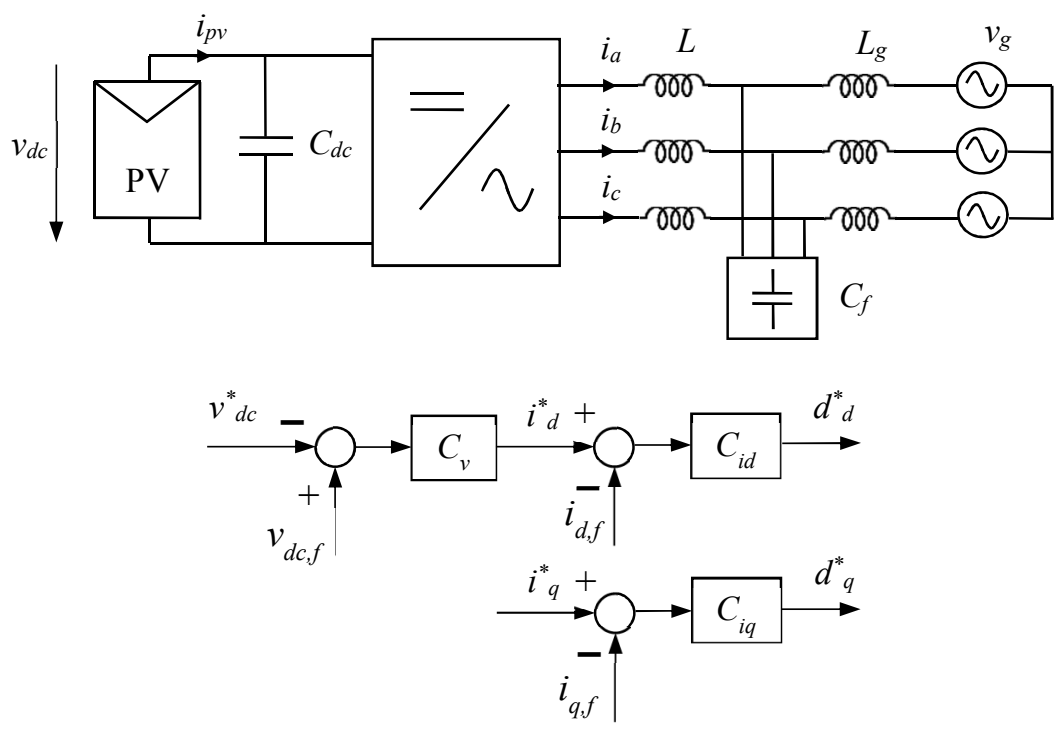

Fig. 1. Three-phase photovoltaic inverter connected to the grid and its control scheme in dq reference frame.

the slope of the I-V curve. Then, the small-signal model is expressed in state-space matrix representation, where the output variables are selected equal to the state variables, i.e. $Y=X$. The state equation can be defined as

$$
\begin{aligned}
& {\left[\begin{array}{c}
\frac{d \hat{i}_{d}}{d t} \\
\frac{d \hat{i}_{q}}{d t} \\
\frac{d \hat{v}_{d c}}{d t}
\end{array}\right]=\left[\begin{array}{ccc}
-\frac{r_{L}}{L} & \omega_{0} & \frac{D_{d}}{L} \\
-\omega_{0} & -\frac{r_{L}}{L} & \frac{D_{q}}{L} \\
-\frac{D_{d}}{C_{d c}} & -\frac{D_{q}}{C_{d c}} & -\frac{g_{p v}}{C_{d c}}
\end{array}\right] \cdot\left[\begin{array}{c}
\hat{i}_{d} \\
\hat{i}_{q} \\
\hat{v}_{d c}
\end{array}\right]+} \\
& +\left[\begin{array}{cccc}
\frac{V_{d c}}{L} & 0 & -\frac{1}{L} & 0 \\
0 & \frac{V_{d c}}{L} & 0 & -\frac{1}{L} \\
-\frac{I_{d}}{C_{d c}} & -\frac{I_{q}}{C_{d c}} & 0 & 0
\end{array}\right] \cdot\left[\begin{array}{c}
\hat{d}_{d} \\
\hat{d}_{q} \\
\hat{v}_{g d} \\
\hat{v}_{g q}
\end{array}\right]
\end{aligned}
$$

From (3) and applying the Laplace transformation, the small-signal control-to-output transfer functions can be obtained. Omitting the grid disturbances, $v_{g d}$ and $v_{g q}$, the corresponding relationships are shown in (4), and the six transfer functions can be consulted in the appendix.

$$
\left\{\begin{array}{l}
\hat{i}_{d}=G_{d d}(s) \cdot \hat{d}_{d}+G_{d q}(s) \cdot \hat{d}_{q} \\
\hat{i}_{q}=G_{q d}(s) \cdot \hat{d}_{d}+G_{q q}(s) \cdot \hat{d}_{q} \\
\hat{v}_{d c}=G_{v d}(s) \cdot \hat{d}_{d}+G_{v q}(s) \cdot \hat{d}_{q}
\end{array}\right.
$$

\section{B. Initial design of the Current Controllers}

As can be observed in Fig. 1, both currents $i_{d}$ and $i_{q}$ are regulated. The d-axis reference is obtained by the outer loop so that the $\mathrm{PV}$ voltage is controlled. The corresponding controller $C_{i d}$ can thus be a $\mathrm{P}$ controller, since a dc current error will be compensated by the voltage controller $C_{v}$. In contrast, the q-axis current loop must track the reference with no dc error so that the reactive power is correctly controlled. As a result, the corresponding controller $C_{i q}$ should include an integral term, and thus a PI controller is selected. These considerations lead to the following current controllers:

$$
C_{i d}(s)=K_{P d}, \quad C_{i q}(s)=K_{P q} \cdot \frac{T_{n} s+1}{T_{n} s},
$$

where $K_{P d}$ and $K_{P q}$ are the proportional gains and $T_{n}$ is the time constant of the PI controller.

The proportional gains will be tuned by using frequencydomain analysis. Although the system is actually MultipleInput Multiple-Output (MIMO), it can be treated as two Single-Input Single-Output (SISO) for high frequencies around the crossover frequency. This leads to the current control loops shown in Fig. 2, where $i^{*}{ }_{d}$ and $i^{*}{ }_{q}$ are the reference currents, $d^{*}{ }_{d}$ and $d^{*}{ }_{q}$ the reference duty cycles, $i_{d, f}$ and $i_{q, f}$ the measured currents, $D_{i}$ represents the computation and zero-order hold delays and $H_{i}$ the current filters.
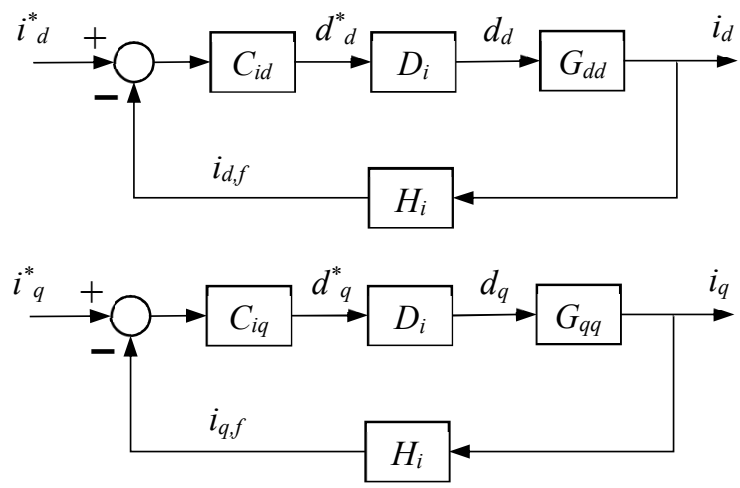

Fig. 2. D- and q-axes current control loops.

From Fig. 2, the compensated open-loop transfer functions for both d-and q-axes can be expressed as

$$
\left\{\begin{array}{l}
O L_{d}(s)=C_{i d} \cdot D_{i} \cdot G_{d d} \cdot H_{i} \\
O L_{q}(s)=C_{i q} \cdot D_{i} \cdot G_{q q} \cdot H_{i}
\end{array}\right.
$$


The plant transfer functions $G_{d d}(\mathrm{~s})$ and $G_{q q}(\mathrm{~s})$, without parasitics and for $I_{q}=0$, are shown in (7) and (8), where $g_{t}=g_{p v}-g_{0}$ is the PV total conductance and $g_{0}=I_{p v} / V_{d c}$ is the PV static conductance. It is worth highlighting that, while $g_{p v}$ and $g_{0}$ are always positive, the total conductance $g_{t}$ is positive when $V_{d c}$ is over the MPP voltage $V_{M P P}$ and negative for $V_{d c}<V_{M P P}$. For the controller design, the high frequency approximation shown in (9) can be applied.

$$
\begin{gathered}
G_{d d}(s)=\frac{V_{d c} s \cdot\left(C_{d c} s+g_{t}\right)}{L C_{d c} s^{3}+L g_{p v} s^{2}+\left(\frac{V_{g d}^{2}}{V_{d c}^{2}}+L C_{d c} \omega_{0}^{2}\right) s+L g_{p v} \omega_{0}^{2}} \\
G_{q q}(s)=\frac{V_{d c} C_{d c} s^{2}+V_{d c} g_{p v} s+\frac{1}{L} \frac{V_{g}^{2}}{V_{p v}}}{L C_{d c} s^{3}+L g_{p v} s^{2}+\left(\frac{V_{g}^{2}}{V_{d c}^{2}}+L C_{d c} \omega_{0}^{2}\right) s+L g_{p v} \omega_{0}^{2}} \\
G_{d d}(s) \approx G_{q q}(s) \approx \frac{V_{d c}}{L \cdot s}
\end{gathered}
$$

Furthermore, if the time constant of the q-axis controller is selected so that $T_{n} \cdot \omega_{c i q} \geq 15$, where $\omega_{c i q}$ is the $\mathrm{q}$ current crossover frequency, the controller $C_{i q}$ resembles a $\mathrm{P}$ controller around $\omega_{\text {ciq. }}$. Taking into account these considerations, the open-loop transfer functions shown in (6) can be approximated as

$$
\left\{\begin{array}{l}
O L_{d_{-} a p r o x}(s)=K_{P d} \cdot e^{-T_{i} \cdot s} \cdot \frac{1-e^{-T_{i} \cdot s}}{T_{i} \cdot s} \cdot \frac{V_{d c}}{L \cdot s} \cdot \frac{1}{s / \omega_{c H i}+1} \\
O L_{q_{-} \text {aprox }}(s)=K_{P q} \cdot e^{-T_{i} \cdot s} \cdot \frac{1-e^{-T_{i} \cdot s}}{T_{i} \cdot s} \cdot \frac{V_{d c}}{L \cdot s} \cdot \frac{1}{s / \omega_{c H i}+1}
\end{array}\right.
$$

where $T_{i}$ is the sampling time, and $\omega_{c H i}$ the current filter cutoff frequency.

Parameters $K_{P d}$ and $K_{P q}$ are designed to have certain crossover frequencies $\omega_{\text {cid }}$ and $\omega_{\text {ciq }}$, respectively. Furthermore, the selected operating point is with $V_{d c}=V_{d c, \max }$, since this point represents the worst conditions concerning stability. Hence, from (10), the proportional gains can be selected as

$$
\left\{\begin{array}{l}
K_{P d}=\frac{L \cdot \omega_{c i d}}{V_{d c, \text { max }}}=\frac{x_{L} \cdot V_{g}^{2}}{V_{d c, \text { max }} \cdot S_{i n v}} \cdot \frac{\omega_{c i d}}{\omega_{0}} \\
K_{P q}=\frac{L \cdot \omega_{c i q}}{V_{d c, \text { max }}}=\frac{x_{L} \cdot V_{g}^{2}}{V_{d c, \text { max }} \cdot S_{i n v}} \cdot \frac{\omega_{c i q}}{\omega_{0}}
\end{array}\right.
$$

where $x_{L}$ is the per-unit impedance of the inductor and $S_{i n v}$ is the inverter nominal power.

Once the crossover frequencies are chosen, the phase margins $P M_{i d}$ and $P M_{i q}$ can be obtained from (10) as

$$
\left\{\begin{array}{l}
P M_{i d}=\frac{\pi}{2}-\frac{3}{2} T_{i} \omega_{c i d}-\arctan \left(\frac{\omega_{c i d}}{\omega_{c H i}}\right) \\
P M_{i q}=\frac{\pi}{2}-\frac{3}{2} T_{i} \omega_{c i q}-\arctan \left(\frac{\omega_{c i q}}{\omega_{c H i}}\right)
\end{array}\right.
$$

For the selection of the crossover frequencies, two restrictions must be taken into account. The first one can be derived from (12) and shows that their maximum value is limited so that a certain phase margin is ensured. The second one is related to the low-frequency RHP pole which appears when operating with $V_{d c}<V_{M P P}$. This condition is only applied to the $\mathrm{d}$ axis and will be described below.

As an example, the current control design is carried out for a system with the parameters shown in Table I, where $f_{s w}$ is the switching frequency and $f_{s}$ is the sampling frequency. In order to have a phase margin $P M_{i d}=P M_{i q}=2 \pi / 9\left(40^{\circ}\right)$, from (12) both crossover frequencies are selected as $f_{\text {cid }}=f_{\text {ciq }}=2000 \mathrm{~Hz}$. Then, from (11), the proportional gains are obtained as $K_{P d}=K_{P q}=10.24 \cdot 10^{-3} \mathrm{~A}^{-1}$. Concerning the time constant $T_{n}$ for the q-axis controller, it is selected so that $T_{n} \cdot \omega_{\text {ciq }}=20$ and thus the phase margin is hardly affected.

TABLE I. SYSTEM PARAMETERS

\begin{tabular}{|c|c|}
\hline Rated values & $S_{i n v}=50 \mathrm{kVA}, f_{0}=50 \mathrm{~Hz}, V_{g}=400 \mathrm{~V}$ \\
\hline DC voltage & $V_{d c, \text { min }}=570 \mathrm{~V}, V_{d c, \text { max }}=1000 \mathrm{~V}$ \\
\hline Input capacitor & $C_{d c}=622 \mu \mathrm{F}$ \\
\hline Inverter inductor & $x_{L}=0.08, L=815 \mu \mathrm{H}, r_{L}=45 \mathrm{~m} \Omega$ \\
\hline PV generator & $g_{t, \text { min }}=-150 \mathrm{mS}, g_{t, \text { max }}=1500 \mathrm{mS}$ \\
\hline Inverter frequencies & $f_{s w}=15 \mathrm{kHz}, f_{s}=30 \mathrm{kHz}, f_{c H i}=8 \mathrm{kHz}$ \\
\hline & $P M_{i d}=P M_{i q}=40^{\circ} \rightarrow f_{c i d}=f_{c i q}=2000 \mathrm{~Hz}$ \\
Control design & $K_{P d}=K_{P q}=10.24 \cdot 10^{-3} \mathrm{~A}^{-1}$ \\
& $T_{n} \cdot \omega_{c i q}=20 \rightarrow T_{n}=1.59 \mathrm{~ms}$ \\
\hline
\end{tabular}

Figure 3 shows the Bode plot of the compensated openloop transfer functions for $V_{d c}=V_{d c \text {, max }}$. The three represented curves are the complete open-loop transfer function for the $\mathrm{d}$ axis, $O L_{d}=C_{i d} \cdot D_{i} \cdot G_{d d} \cdot H_{i}$, the complete open-loop transfer function for the q axis, $O L_{q}=C_{i d} \cdot D_{i} \cdot G_{q q} \cdot H_{i}$, and the open-loop transfer function $O L=O L_{d \_a p r o x}=O L_{q_{a} \text { aprox }}$, approximated as shown in (10). As can be observed, the three plots are almost identical at high frequencies and as a result the crossover frequencies and phase margins are close to their target values, which justifies the use of (10)-(12) for the controller design.

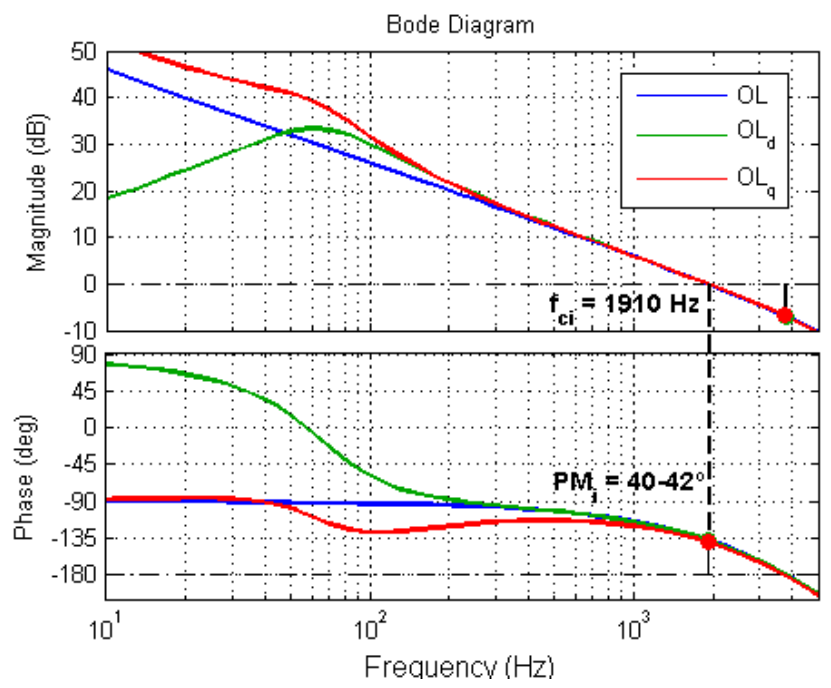

Fig. 3. Compensated open-loop transfer functions for $V_{d c}=V_{d c \text {, } m a x}$.

Finally, once the current controls have been implemented, closed-loop transfer functions $G_{c l}$ can be defined so that 


$$
\left\{\begin{array}{l}
\hat{i}_{d}=G_{c l, d d}(s) \cdot \hat{i}_{d}^{*}+G_{c l, d q}(s) \cdot \hat{i}_{q}^{*} \\
\hat{i}_{q}=G_{c l, q d}(s) \cdot \hat{i}_{d}^{*}+G_{c l, q q}(s) \cdot \hat{i}_{q}^{*} \\
\hat{v}_{d c}=G_{c l, v d}(s) \cdot \hat{i}_{d}^{*}+G_{c l, v q}(s) \cdot \hat{i}_{q}^{*}
\end{array}\right.
$$

\section{Open-Loop Transfer Function of the Voltage Regulation}

The voltage control loop is shown in Fig. 4, where $v_{d c}^{*}$ is the reference PV voltage, $v_{d c, f}$ the measured voltage, $C_{v}$ represents the PI controller, $D_{v}$ the computation and zeroorder hold delays and $H_{v}$ the voltage filter.

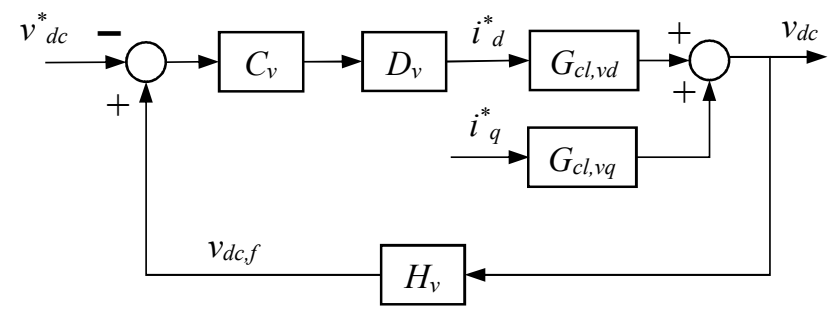

Fig. 4. PV voltage control loop.

To design the voltage controller, a model of the transfer function $G_{c l, v d}$, valid at low frequencies, must be first obtained. For this purpose, processes which occur at high frequencies, such as the computation and zero-order hold delays $D_{i}$, and the current filter $H_{i}$, can be disregarded. Figure 5 shows two equivalent schemes which make it possible to obtain $G_{c l, v d}$ expression, where Figure 5(a) employs the currents $i_{d}$ and $i_{q}$ as intermediate variables and will be used for the ideal case, and Figure 5(b) employs the duty cycles $d_{d}$ and $d_{q}$ as intermediate variables and will be used for the real case.

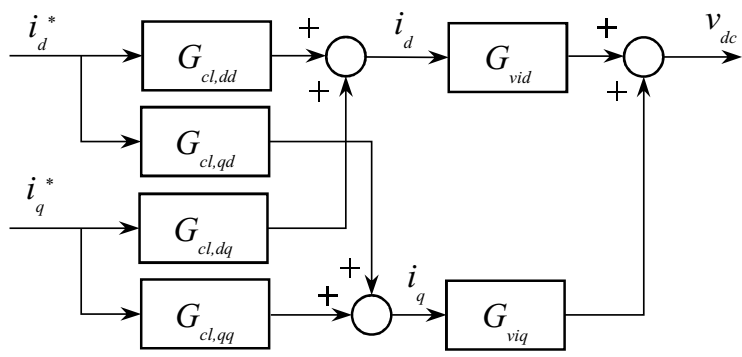

(a) Scheme used for the ideal case

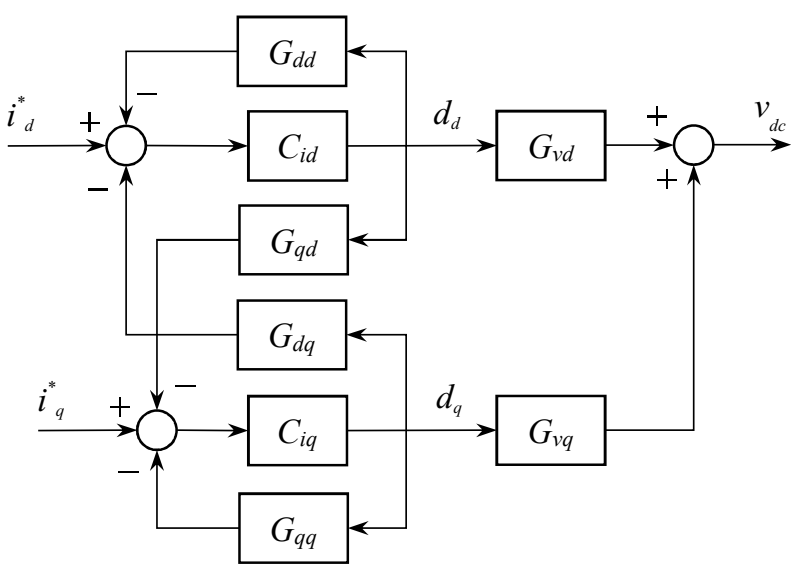

(b) Scheme used for the real case

Fig. 5. Schemes used to obtain the closed-loop transfer function $G_{c l, v d \text {. }}$
From Fig. 5(a), the transfer function $G_{c l, v d}$ can be obtained as shown in (14). If a fast PI controller is used in both $d$ and $q$ axes, the current loop can be taken as ideal at low-frequencies, that is $G_{c l, d d}=1$ and $G_{c l, q d}=0$, leading to the approximation expressed in (15). As can be observed, in this case the closedloop transfer function presents a pole at $\mathrm{s}=-g_{t} / C_{d c}$, which is at the RHP when $g_{t}$ is negative, i.e. when the voltage is below the MPP voltage and the slope of the P-V curve is positive. In order to reduce this pole frequency and allow for a stable voltage control, the input capacitor must be oversized [5].

$$
\begin{aligned}
& G_{c l, v d}(s)=\frac{\hat{v}_{d c}}{\hat{i}_{d}^{*}}=G_{c l, d d} \cdot G_{v i d}+G_{c l, q d} \cdot G_{v i q} \\
& G_{c l, v d}(s) \approx G_{v i d}(s)=\frac{\hat{v}_{d c}}{\hat{i}_{d}} \approx-\frac{V_{g} / V_{d c}}{C_{d c} \cdot s+g_{t}}
\end{aligned}
$$

However, when a $\mathrm{P}$ controller is used as regulator $C_{i d}$, the ideal current loop assumption, usually made in the literature, is no longer valid. Furthermore, the model must consider a MIMO system. In so doing, from Fig. 5(b), transfer function $G_{c l, v d}$ can be expressed as shown in (16). This expression can be approximated as the simple first-order transfer function shown in (17) and (18). This approximation is derived in the appendix and reproduces accurately the closed-loop lowfrequency behaviour.

As can be observed, (17) is very similar to (15), except that using a $\mathrm{P}$ controller in the d-axis transforms the real capacitance $C_{d c}$ and the real conductance $g_{t}$ into an equivalent capacitance $C_{e q}$ and an equivalent conductance $g_{\text {teq }}$. In practice, the effect of the current closed-loop is a slight modification of the dc capacitance but an important increase of the total conductance. Since the closed-loop pole is now located at $\mathrm{s}=-g_{t e q} / C_{e q}$ instead of $\mathrm{s}=-g_{t} / C_{d c}$, the frequency of the pole is reduced, thus moving towards the LHP for $g_{t}<0$. It is worth noting that the values of $C_{e q}$ and $g_{t e q}$ are affected by the d-axis controller $C_{i d}$ but are totally independent of the qaxis controller $C_{i q}$.

$$
\begin{gathered}
G_{c l, v d}(s)=\frac{C_{i d} G_{v d}+C_{i d} C_{i q}\left(G_{v d} G_{q q}-G_{v q} G_{q d}\right)}{1+C_{i d} G_{d d}+C_{i q} G_{q q}+C_{i d} C_{i q}\left(G_{d d} G_{q q}-G_{d q} G_{q d}\right)} \\
G_{c l, v d}(s) \approx-\frac{V_{g} / V_{d c}}{C_{e q} \cdot s+g_{t e q}} \\
C_{e q}=C_{d c}+\frac{L \cdot g_{p v}}{V_{d c}} \cdot \frac{1}{K_{P d}}, \quad g_{t e q}=g_{t}+\frac{V_{g}^{2}}{V_{d c}^{3}} \cdot \frac{1}{K_{P d}}
\end{gathered}
$$

\section{Final Design of the Current Controllers}

Concerning stability, the most critical operating point is with $V_{d c}=V_{d c \text {, min }}$, where the total conductance is minimum, $g_{t}=g_{t, m i n}$, and $g_{p v} \approx 0$. At this point, from (18) and considering $V_{d c, \min } \approx \sqrt{2} \cdot V_{g}$, one obtains

$$
C_{e q} \approx C_{d c}, \quad g_{t e q, \min } \approx g_{t, \text { min }}+\frac{1}{2 V_{d c, \text { min }}} \cdot \frac{1}{K_{P d}}
$$

The minimum total conductance can be estimated as [3] 


$$
g_{t, \min }=-\frac{S_{i n v}}{V_{d c, \min }^{2}}
$$

Then, substituting $K_{P d}$ and $g_{t, \min }$ expressions, (11) and (20), into (19) leads to another expression for the minimum value of $g_{\text {teq }}$, that is

$$
g_{\text {teq, } \min } \approx \frac{S_{i n v}}{V_{d c, \text { min }}^{2}} \cdot\left(\frac{V_{d c, \text { max }}}{V_{d c, \text { min }}} \cdot \frac{\omega_{0}}{x_{L} \omega_{c i d}}-1\right)
$$

In a similar way, if the input capacitance is defined as a function of its per-unit impedance, $x_{C}$, it can be expressed as

$$
C_{e q} \approx C_{d c}=\frac{1}{x_{C} \omega_{0}} \cdot \frac{S_{i n v}}{V_{g}^{2}} \approx \frac{2}{x_{C} \omega_{0}} \cdot \frac{S_{i n v}}{V_{d c, \text { min }}^{2}}
$$

Thus, from (21) and (22), the maximum frequency of the closed-loop pole can be obtained as

$$
\omega_{p, \text { max }}=-\frac{g_{\text {teq, } \min }}{C_{e q}} \approx \frac{x_{C} \omega_{0}}{2} \cdot\left(1-\frac{V_{d c, \text { max }}}{V_{d c, \text { min }}} \cdot \frac{\omega_{0}}{x_{L} \omega_{c i d}}\right)
$$

As can be observed, the frequency of this pole depends on the crossover frequency selected for the d current control. In this paper, it is first proposed to restrict its value so that $\omega_{p, \max }=0$ and thus the RHP pole is avoided in the whole operation range. From (23), one obtains

$$
\omega_{c i d}=\frac{V_{d c, \text { max }}}{V_{d c, \text { min }}} \cdot \frac{\omega_{0}}{x_{L}} \Rightarrow g_{\text {teq, } \text { min }}=0, \omega_{p, \text { max }}=0
$$

As a result, considering $V_{d c \text {, min }} \approx \sqrt{2} \cdot V_{g},(17)$ and (24), the transfer function $G_{c l, v d}$ then becomes

$$
G_{c l, v d}(s) \approx-\frac{1 / \sqrt{2}}{C_{d c} \cdot s}
$$

An alternative is to choose a higher $\omega_{\text {cid }}$, which makes that the current closed-loop becomes unstable in the worst operating point, but limiting the frequency of the pole to $\omega_{c v} / 2$, where $\omega_{c v}$ is the crossover frequency of the PV voltage control. In this way, the outer loop will be able to stabilize the control. From (23), the crossover frequency should then be selected as

$$
\omega_{c i d}=\frac{V_{d c, \max }}{V_{d c, \min }} \cdot \frac{\omega_{0}}{x_{L}} \cdot \frac{1}{1-\frac{\omega_{c v}}{x_{C} \omega_{0}}} \Rightarrow \omega_{p, \max }=\frac{\omega_{c v}}{2}
$$

Figure 6 shows the frequency of the dominant pole at $V_{d c}=V_{d c, \min }$, as a function of the crossover frequency $f_{c i d}$, for the system parameters presented in Table I. As can be observed, the pole frequency obtained with the first-order approximation, as expressed in (23), is very similar to the exact value, obtained with a complete MIMO model which takes into account all dynamics. If the crossover frequency is selected equal to $2000 \mathrm{~Hz}$, so that the phase margin is $40^{\circ}$ (see section II.B), the dominant pole is located at $+18.1 \mathrm{~Hz}$. As a result, it is not possible to stabilize the system without increasing the input capacitance. However, if the crossover frequency is selected equal to $1100 \mathrm{~Hz}$ following (24), the dominant pole is located at the origin. Finally, choosing the crossover frequency equal to $1375 \mathrm{~Hz}$ according to (26), the dominant pole is located at $f_{c v} / 2=+7.5 \mathrm{~Hz}$. The two latter situations make it possible to design a stable outer loop without requiring an increase of the input capacitance.

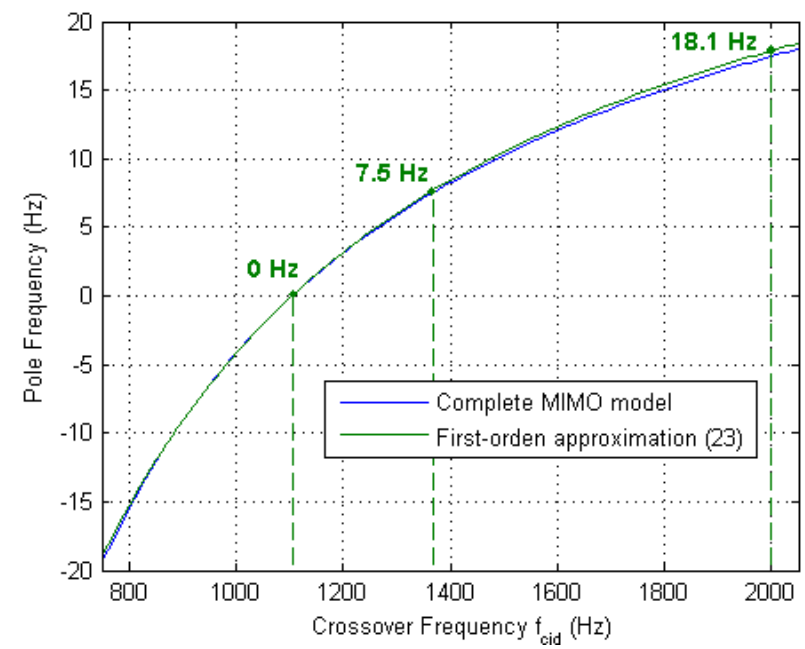

Fig. 6. Dominant pole of the current closed-loop at $V_{d c}=V_{d c, m i n}$, as a function of the crossover frequency.

\section{SiMULATION RESULTS}

The proposed method is validated with the PSIM software for the system with parameters shown in Table I. The voltage control is tested for three different d-axis current controllers: (P1) for a $\mathrm{P}$ controller designed as shown in section II.B, i.e. to have maximum crossover frequency, $f_{\text {cid }}=2000 \mathrm{~Hz}$; (P2) for a $\mathrm{P}$ controller designed as proposed in this paper to

\begin{tabular}{|c|c|}
\hline $\begin{array}{l}\text { P controller for } \\
\text { maximum } f_{\text {cid }}\end{array}$ & $\begin{array}{c}P M_{i d}=40^{\circ} \rightarrow f_{c i d}=2000 \mathrm{~Hz} \\
K_{P d}=10.24 \cdot 10^{-3} \mathrm{~A}^{-1}\end{array}$ \\
\hline $\begin{array}{l}\text { P controller to avoid } \\
\text { the RHP pole }\end{array}$ & $\begin{array}{c}\omega_{p, \max }=0 \rightarrow f_{c i d}=1100 \mathrm{~Hz} \\
K_{P d}=5.63 \cdot 10^{-3} \mathrm{~A}^{-1}, P M_{i}=62.2^{\circ}\end{array}$ \\
\hline PI controller & $\begin{array}{c}P M_{i d}=40^{\circ}, f_{\text {cid }}=1100 \mathrm{~Hz} \\
K_{P d}=5.21 \cdot 10^{-3} \mathrm{~A}^{-1}, T_{n d}=0.35 \mathrm{~ms}\end{array}$ \\
\hline Voltage loop & $f_{c v}=15 \mathrm{~Hz}, P M_{v}=45^{\circ}$ \\
\hline
\end{tabular}
avoid the RHP pole, i.e. with $f_{\text {cid }}=1100 \mathrm{~Hz}$ from (24); and (PI) for a PI controller designed to have $f_{\text {cid }}=1100 \mathrm{~Hz}$ and phase margin $P M_{i d}=40^{\circ}$. These specifications as well as the ones applied to design the voltage controller are included in Table II.

TABLE II. SYSTEM PARAMETERS

The results are shown in Fig. 7 for steps in the voltage reference near and below the MPP voltage $\left(V_{M P P}=628 \mathrm{~V}\right)$, where the following variables are represented: dc voltage, reference dc voltage, $\mathrm{d}$-axis current and PV total conductance $g_{t}$. As can be observed, the response for the P1 controller becomes oscillating at low voltage [Fig. 7(a)], while the proposed $\mathrm{P} 2$ controller ensures stability in the whole operating range, including with a total conductance as low as $g_{t}=-120 \mathrm{mS}$ [Fig. 7(b)]. Finally, the response for the PI controller becomes unstable below MPP voltage [Fig. 7(c)]. 

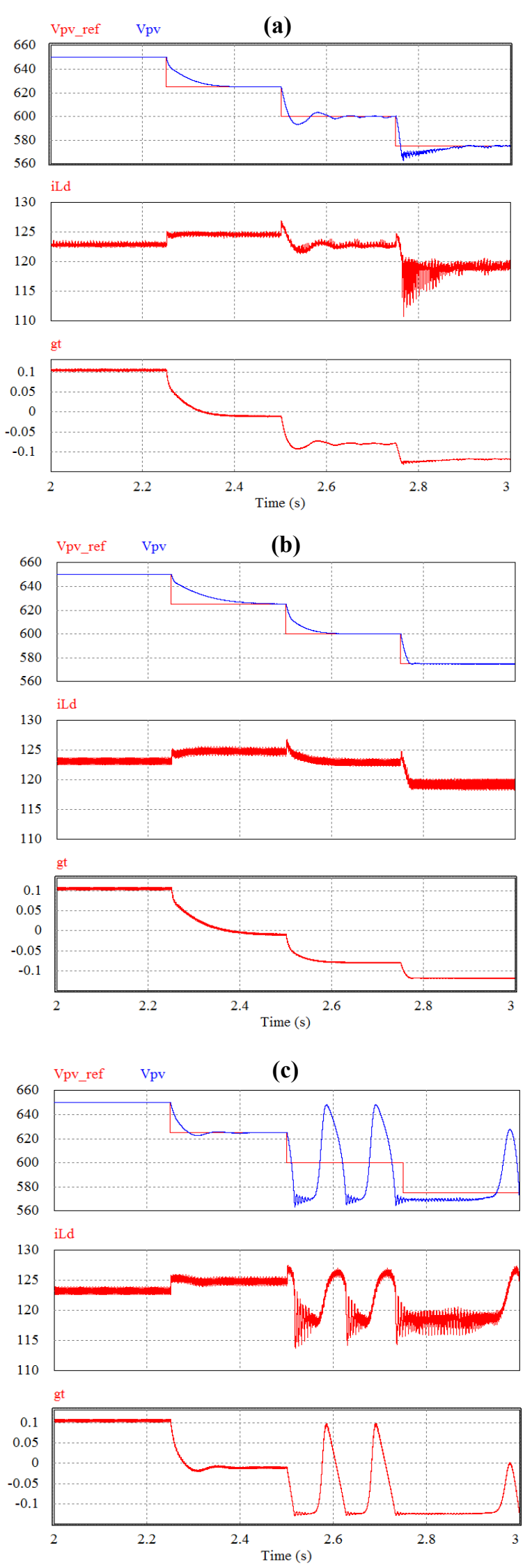

Fig. 7. Simulation results: (a) $\mathrm{P}$ controller designed for $f_{\text {cid }}=2000 \mathrm{~Hz}$, (b) $\mathrm{P}$ controller designed for $f_{\text {cid }}=1100 \mathrm{~Hz}$, (c) PI controller designed for $f_{c i d}=1100 \mathrm{~Hz}$ and $P M_{i d}=40^{\circ}$.

\section{CONCLUSION}

This paper analyzes the voltage regulation in gridconnected three-phase PV inverter, paying attention to the effect of the inner current loop. When using a PI controller, at low-frequencies the current loop can be considered as ideal and the inverter as a constant power load, which exposes the system RHP pole and forces to increase the input capacitance. On the other hand, using in the d-axis a $\mathrm{P}$ controller with restricted bandwidth reduces the frequency of the RHP pole, permitting to obtain an adequate voltage regulation in the whole operating range with no need of oversizing the capacitance.

\section{APPENDIX}

This appendix explains how to obtain the first-order approximation shown in (17) on the basis of (16). First of all, the control-to-output transfer function, defined by (4), without parasitics and for $I_{q}=0$, can be expressed as

$$
\begin{gathered}
G_{d d}(s)=\frac{V_{d c} s \cdot\left(C_{d c} s+g_{t}\right)}{d(s)} \\
G_{q q}(s)=\frac{V_{d c} C_{d c} s^{2}+V_{d c} g_{p v} s+\frac{1}{L} \frac{V_{g}^{2}}{V_{p v}}}{d(s)} \\
G_{d q}(s)=\frac{V_{d c} \omega_{0} \cdot\left(C_{d c} s+g_{t}\right)}{d(s)} \\
G_{q d}(s)=-\frac{V_{d c} \omega_{0} \cdot\left(C_{d c} s+g_{p v}\right)}{d(s)} \\
G_{v d}(s)=-\frac{V_{g} s}{d(s)} \\
G_{v q}(s)=-\frac{V_{g} \omega_{0}}{d(s)} \\
d(s)=L C_{d c} s^{3}+L g_{p v} s^{2}+\left(\frac{V_{g}^{2}}{V_{d c}^{2}}+L C_{d c} \omega_{0}^{2}\right) s+L g_{p v} \omega_{0}^{2}
\end{gathered}
$$

In addition, from (A.1)-(A.7), the following expressions can be obtained:

$$
\begin{gathered}
G_{v d} \cdot G_{q q}-G_{v q} \cdot G_{q d}=-\frac{V_{g} V_{d c}}{L} \cdot \frac{1}{d(s)} \\
G_{d d} \cdot G_{q q}-G_{d q} \cdot G_{q d}=\frac{V_{d c}^{2}}{L} \cdot \frac{C_{d c} s+g_{t}}{d(s)}
\end{gathered}
$$

From (16), at low frequencies the term 1 in the denominator is much lower than the other term. As a result, it can be neglected, which results in

$$
G_{c l, v d}(s)=\frac{G_{v d}+C_{i q}\left(G_{v d} G_{q q}-G_{v q} G_{q d}\right)}{G_{d d}+\frac{C_{i q}}{C_{i d}} G_{q q}+C_{i q}\left(G_{d d} G_{q q}-G_{d q} G_{q d}\right)}
$$


Substituting the corresponding expressions (5), (A.1), (A.2), (A.5), (A.8) and (A.9) into (A.10), and reorganizing, leads to

$$
G_{c l, v d}(s) \approx-\frac{V_{g d}}{V_{d c}} \cdot \frac{a_{2} s^{2}+a_{1} s+1}{b_{3} s^{3}+b_{2} s^{2}+b_{1} s+b_{0}},
$$

where

$$
\left\{\begin{array}{l}
a_{2}=\frac{L}{V_{d c}} \cdot \frac{T_{n}}{K_{P q}}, \quad a_{1}=T_{n} \\
b_{3}=\frac{L C_{d c}}{V_{d c}} \cdot T_{n}\left(\frac{1}{K_{P d}}+\frac{1}{K_{P q}}\right) \\
b_{2}=\frac{L g_{p v}}{V_{d c}} \cdot \frac{T_{n}}{K_{P d}}+\frac{L g_{t}}{V_{d c}} \cdot \frac{T_{n}}{K_{P q}}+\frac{L C_{d c}}{V_{d c}} \cdot \frac{1}{K_{P d}}+T_{n} C_{d c} \\
b_{1}=C_{d c}+\frac{L g_{p v}}{V_{d c}} \cdot \frac{1}{K_{P d}}+\frac{V_{g}^{2}}{V_{d c}^{3}} \cdot \frac{T_{n}}{K_{P d}}+T_{n} g_{t} \\
b_{0}=g_{t}+\frac{V_{g}^{2}}{V_{d c}^{3}} \cdot \frac{1}{K_{P d}}
\end{array}\right.
$$

At low frequencies, $G_{c l, v d}(\mathrm{~s})$ is determined by one dominant pole. From (A.11) and (A.12), the Padé approximant of order $[0 / 1]$ can be obtained as

$$
\left\{\begin{array}{l}
G_{c l, v d}(s) \approx-\frac{V_{g}}{V_{d c}} \cdot \frac{1}{C_{e q} s+g_{\text {teq }}} \\
g_{\text {teq }}=b_{0}=g_{t}+\frac{V_{g}^{2}}{V_{d c}^{3}} \cdot \frac{1}{K_{P d}} \\
C_{e q}=b_{1}-b_{0} \cdot a_{1}=C_{d c}+\frac{L \cdot g_{p v}}{V_{d c}} \cdot \frac{1}{K_{P d}}
\end{array}\right.
$$

\section{REFERENCES}

[1] J. Samanes, A. Urtasun, E. Gubia, and A. Petri, "Robust multisampled capacitor voltage active damping for grid-connected power converters," Electrical Power and Energy Systems, vol. 105, pp. 741$752,2019$.

[2] T. Messo, J. Jokipii, and T. Suntio, "Minimum dc-link capacitance requirement of a two-stage photovoltaic inverter," in 2013 IEEE Energy Conversion Congress and Exposition, pp. 999-1006, 2013.

[3] A. Urtasun, P.Sanchis, and L. Marroyo, "DC capacitance reduction in three-phase photovoltaic inverters by using virtual impedance emulation," in $21^{s t}$ European Conference on Power Electronics and Applications (EPE-ECCE), pp. 1-10, 2019.

[4] S. Skogestad, I. Postlethwaite, "Multivariable feedback control: Analysis and design," John Wiley \& Sons Ltd., West Sussex, England, 1996.

[5] T. Messo, J. Jokipii, J. Puukko, and T. Suntio, "Determining the value of dc-link capacitance to ensure stable operation of a three-phase photovoltaic inverter," IEEE Transactions on Power Electronics, vol. 29, no. 2, pp. 665-673, 2014. 